\title{
Inhibitor performance and the preparation study of urea formaldehyde resin matrix composite particle
}

\author{
LIN Shao-sen ${ }^{a}$, YAN Jun ${ }^{b}$, LI Hong-guang ${ }^{c}$, DU Shi-guo ${ }^{b}$ \\ Ordnance Engineering College, Shijiazhuang 050003, Hebei, China \\ aIss_xuqing@163.com, byan-junjun@263.net, 'cqdlhgcy6@126.com
}

Keywords: inhibitor; metatitanic acid; urea-formaldehyde resin; composite particle

\begin{abstract}
In order to improve the service life of weapons, an erosion additives of urea formaldehyde(UF) resin matrix composite particle is prepared by using in-situ polymerization method. The morphology composite particle is represented through scanning electron microscope, and the thermal analysis method is utilized to determine the organic and inorganic components proportion of erosion inhibitors. Finally, a contrasting ablation tube test is made to test its inhibitor performance. The experimental results show that the prepared inhibitor has a good particle dispersion and uniformity. The allocation ratio of organic and inorganic components is about 5.38:1. Compared to erosion inhibitor efficiency of standard propellant, that of the prepared erosion inhibitor can reach $17.56 \%$. The application of this type of erosion inhibitor can decrease the ablative properties of the propellant, which is expected to reduce the erosion problems of high energy propellant.
\end{abstract}

\section{Introduction}

While artillery achieve long-range, high velocity and rapid fire, environment of gun tube with high temperature, high pressure and high loading dose makes barrel erosion problems become increasingly prominent. To inhibit gun tube wear and erosion, domestic and foreign scholars have proposed many possible methods including the coatings of inner bore of barrel, optimization of shape and cartridge belt material of artillery, manufacturing technology of composite pipe, cooling technology of barrel, barrel self-repair technology, low temperature propellant and inhibitor additives technology[1-8]. Among them, the inhibitor additives technology, a kind of simple and effective method, has caught more and more attention.

Inhibitors currently used mainly have $\mathrm{TiO}_{2}$ type, talcum powder-type, multi-type, etc. It is found in the practical application these corrosion inhibitors have instable disadvantages with large dosage in use, particularly powder-loading of high-pressure artillery lacks effective corrosion inhibitor. Beijing Institute of Technology replace a certain inhibitor of gun powder with nano-composite, compare ablation situation before and after the replacement, it is found well-dispersed nanocomposite can play a inhibition effect[5]. Boncompain by tests on 105mm HE C132 artillery, found main components are talc / paraffin, $\mathrm{TiO}_{2}$ / paraffin, polyurethane foam inhibitors, and found Akers Krutbruk product whose main composition is TiO2 / paraffin has best inhibitor effect[6]. Zhengshuang and others prepared an organic silicone corrosion inhibitors, compare ablation properties of organic silicone corrosion inhibitors with different amount and two kinds of propellant with a certain amount of talc and titanium dioxide, and it is found that the organic silicone ablative agent has a better effect of reducing the ablation[7].

Based on problems of traditional corrosion inhibitors and combined with some current design concepts, design better inhibitors, overcome the shortcomings of traditional corrosion inhibitors. It can play a more significant inhibition effect in barrel corrosion, which is of great economic and military value. Combining discussion on the theory of inhibitors by the research group[9], we design a powdered corrosion inhibition with inorganic / organic core-shell structure, use emission environment to form methods of inorganic insulation materials and endothermic decomposition of organic materials for protecting inner bore of barrel. It characterizes morphology and structure of 
composite particles and use ablation tube test method to measure the corrosion performance of core-shell particles.

\section{Experimental preparation}

Materials. Analytic grade urea and formalin were used for synthesis of composite particle.The aqueous solutions of both hydrochloric acid $(0.1 \mathrm{~mol} / \mathrm{L})$ and sodium hydroxide $(0.1 \mathrm{~mol} / \mathrm{L})$ were used to adjust the $\mathrm{PH}$ level during the composite particle synthesis. Sodium dodecyl benzene sulfonate (SDBS) (CP) was used as a modifier. Ammonium chloride ( $\mathrm{NH} 4 \mathrm{Cl}$ ) of $10 \%$ aqueous solution was used as a hardener. The whole experimental process using deionized water.

Preparation of UF resin matrix composite particles. Take $1.51 \mathrm{~g}$ metatitanic acid, $0.98 \mathrm{~g}$ resorcinol, add the appropriate amount of sodium dodecyl benzene sulfonate to make even under stirring state, mix into a milky white suspension in ultrasound system. Place mixing system in a water bath at $25{ }^{\circ} \mathrm{C}$, start to heat, stir at a rate of 700r / min, gradually add $10.36 \mathrm{~g}$ urea, $25.0 \mathrm{~g}$ formaldehyde solution, adjust $\mathrm{PH}=3$ with hydrochloric acid and heat at a rate of $2{ }^{\circ} \mathrm{C} / \mathrm{min}$. When the temperature reaches $45{ }^{\circ} \mathrm{C}$, join $2.5 \mathrm{ml} 10 \mathrm{wt} \%$ ammonium chloride aqueous solution in batches, when the temperature reaches $55{ }^{\circ} \mathrm{C}$ stop heating, and keep warm for $2 \mathrm{~h}$. The microcapsule is filtered with suspension, centrifuged, and washed with ethanol, then dried in $60^{\circ} \mathrm{C}$ vacuum fro $10 \mathrm{H}$. At last, collect powder samples.

With the same method, air load particles can be obtained without adding metatitanic, which can work as comparative sample.

The surface morphology of the prepared inhibitor was studied by SEM (HITACHI-S3400N Japan); Thermal analysis was carried out with a TGA 7 thermogravimetric analyzer(PerkinElmer companies in the United States). Samples (5.5 $\pm 0.2 \mathrm{mg}$ ) were placed in alumina crucibles. An empty alumina crucible was used as reference. Samples were heated from ambient temperature to $600^{\circ} \mathrm{C}$ in a $50 \mathrm{~mL}$ $\min ^{-1}$ flow of $\mathrm{N}_{2}$.

\section{Study on composite particle profile and group assignments ratio}

SEM results and analysis. (a) (d) in Figure 2 are respectively SEM images of composite particles and metatitanic acid before and after calcining. As can be seen in image (a), the prepared metatitanic acid/UF resin composite particles have good uniformity and dispersion, and basic particle size is about $10 \mu \mathrm{m}$. Image (b) is the SEM image of raw material metatitanic acid, it can be seen from the image, the metatitanic acid used has smaller particle size, Although there is a certain degree of agglomeration, but steps in preparing metatitanic acid suspension contain ultrasound dispersion[10-11], so the raw material can be used directly. Compare SEM images of composite particles and metatitanic acid after calcining for $10 \mathrm{~min}$ at $800{ }^{\circ} \mathrm{C}$, it can be seen from image (d) metatitanic acid remains original granular after static calcining, and there is no significant change. However, it is found by analyzing calcination results of metatitanic acid after coated UF resin sample in image (c), the product looks like fine powder, so we presume the composite particles are expected to disperse by means of pressure of gunpowder gas in emission environment of inner bore, which finally protect the inner bore. 

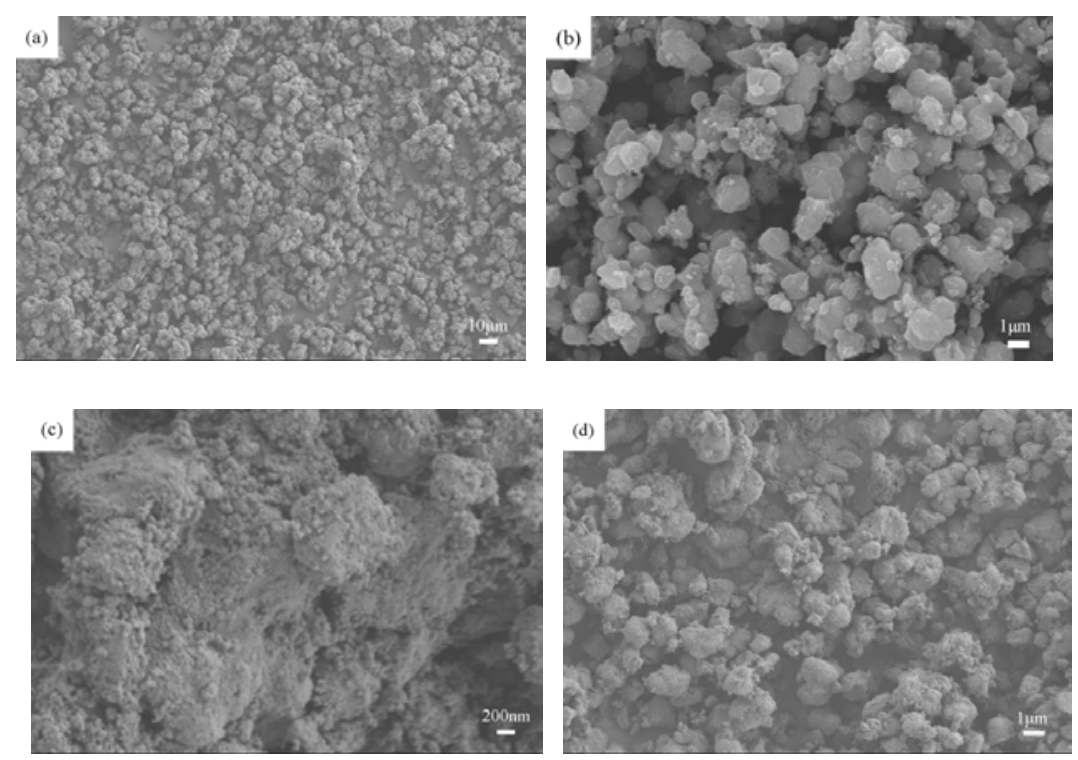

Fig.1 Comparison of composite particles and partial titanate before and after calcination

TGA of composite particles. Fig. 2 is core-shell particles' TG/DTG test results of metatitanic acid/UF resin, weight loss can be divided into room temperature to $220{ }^{\circ} \mathrm{C}, 220 \sim 350{ }^{\circ} \mathrm{C}, 350 \sim$ $600{ }^{\circ} \mathrm{C}$. weight loss in the first phase is about $8 \%$, mainly caused by heated water, ethanol and residual formaldehyde and ammonia gas physically adsorbed[12]. The weight loss in second phase is about $72.2 \%$, and this stage includes methylene ether link made of UF resin shell material, methylene rupture and dehydration of metatitanic acid particles after rupture of core-shell particles. Rupture of methylene ether link corresponds to two peaks in $260{ }^{\circ} \mathrm{C}$ and $279{ }^{\circ} \mathrm{C}$ in image DTG, and methylene corresponds to peak in $305{ }^{\circ} \mathrm{C}$ in DTG. Weight loss is about $7 \%$ in the third phase, and it mainly takes place co-carbonization reaction to remove $\mathrm{N}, \mathrm{H}, \mathrm{O}$ and other elements[13]. The remaining part is mainly $\mathrm{TiO}_{2}$, and $\mathrm{TiO}_{2}$ content available is about $12.8 \%$ which can be converted to about $15.68 \%$ metatitanic acid of the composite particles so that organic component of composite particles is about $84.32 \%$, so the organic and inorganic ratio of composite particles is 5.38:1.

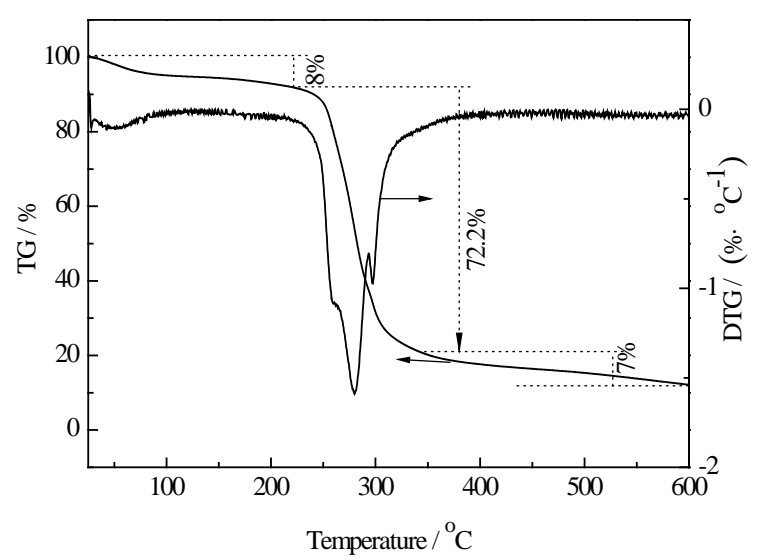

Fig. 2 TG/DTG curve of composite particles

\section{Ablation-resistance performance analysis on composite particles assignments ratio}

Effectiveness evaluation on corrosion inhibitor of metatitanic acid/UF resin. Use ablation tube test method to test performance of ablation-reduction performance of composite particles, we calculate and get 9.7325g loading dose by using energy method, ablation tube material is 45 \# steel, combustion environment is the semi-closed bomb, the control tablet is copper sheet, and ignition power is 2 \# nitrocellulose. Selected propellant is a double vinegar powder. Each sample test three ablation tubes (three ablation tubes' initial quality differential of same tube is $\leqslant 0.005 \mathrm{~g}$ ), and each 
ablation tube test three rounds. Sustained release efficiency is measured by ablation tube weight loss with added inhibitor with respect to powder ablation tube weight loss. Calculation method is as followed[14-15]:

$$
\begin{gathered}
E_{i}=m_{1}-m_{2} \\
E=\frac{\sum_{i=1}^{3} E_{i}}{3} \\
\delta=\frac{\sum_{i=1}^{n}\left|E_{i}-E_{0}\right|}{n} \times 100 \%
\end{gathered}
$$

In the formula: $E_{i}$ is ablation amount of each ablation tube, $i=1,2,3, E$ is ablation amount of each sample; $E_{0}$ is ablative amount of powder, $\delta$ is used to characterize the relative efficiency of sustained release relative to the powder.

Experimental results of erosion tube. By comparing the experimental ablation results of core-shell particles, UF resins and metatitanic acid, it is found metatitanic and urea resins each have a certain corrosion inhibition performance, and the ablation resistance is greatly improved after compositing two, it is believed pure UF resin only can play a cooling effect, but can not protect the barrel bore well. Although the meta-titanic acid alone can protect inner bore, but under the conditions that there is no coated shell material, it can not achieve the expected inflation dispersion effect. Only organic cooling and inorganic effective dispersion can protect inner bore and have ideal protection effect.

Table 1 the result of erosion tube test

\begin{tabular}{cccccc}
\hline \multirow{2}{*}{ Inhibitor } & \multirow{2}{*}{$\begin{array}{c}\text { Mass of } \\
\text { additives(g) }\end{array}$} & Initial mass & Final mass & $\begin{array}{c}\text { Loss of } \\
\text { weight }\end{array}$ & $\begin{array}{c}\text { Effencicy of } \\
\text { inhibitor }\end{array}$ \\
\cline { 3 - 5 } & 0 & 11.5144 & 10.5572 & 0.9572 & \\
Composite particle & 0.15 & 11.5187 & 10.7296 & 0.7891 & $17.56 \%$ \\
UF resin & 0.15 & 11.5330 & 10.6639 & 0.8692 & $9.2 \%$ \\
metatitanic acid & 0.15 & 11.5404 & 10.6178 & 0.9226 & $3.6 \%$ \\
\hline
\end{tabular}

\section{Summary}

1) In the metatitanic acid suspension, the composite particle with diameter of about $10 \mu \mathrm{m}$ was prepared by interfacial polymerization, and the organic and inorganic ratio of composite particles is about 5.38:1.

2) Compare the calcining results before and after coating, it is found the composite particles is expected to further refine and effectively dispersed in the emission conditions, playing the role of protecting the inner bore of barrel.

3) Meta-titanic acid / UF resin composite particles is an effective inhibitor to gun tube, under 1.5 wt\% propellant condition, the inhibition efficiency is up to $17.56 \%$.

\section{References}

[1] Jaramaz S, Micković D, Elek P. Determination of gun propellants erosivity: Experimental and theoretical studies[J]. Experimental Thermal and Fluid Science, 2010, 34(6): 760-765.

[2] Johnston I A. Understanding and predicting gun barrel erosion[R]. Defence Science and Technology Organisation Edinburgh (Australia) Weapons Systems Div, 2005.

[3] Levy R A. Investigation of Chemically Vapor Deposited Tantalum for Medium Caliber Gun Barrel Protection[R]. New Jersey Inst of Tech Newark, 2008. 
[4] de Rosset W S, Montgomery J S. Cobalt-base alloy gun barrel study[J]. Wear, 2014, 316(1): 119-123.

[5] Song Q Z, Duo Y X. Influence of a nanomaterial on propellant and the function of a nanomaterial for increasing the service life of a gun barrel[J]. Journal of Harbin Engineering University, 2011, 32(9): 1173-1178.

[6] Boncompain T. Gun tube wear reduction for $105 \mathrm{~mm}$ artillery[R]. Canada: Ordnance and Tactical Systems, 2010.

[7] Zheng S, Liu B, Liu S W. Application of a new agent of low erosion in the small caliber weapon[J]. Chinese Journal of Energetic Materials, 2011, 19(3): 335-338.

[8] Lin S S, Yan J, Yu W B, et al. The Research Status of Gun Barrel Erosion and Inhibitor Mitigation Mechanism [J]. Journal of Gun Launch \& Control, 2016, 37(1), 92-96.

[9] Zhang Y, Yang C, Zheng J, et al. Crystallization behavior of stable urea formaldehyde resin dispersed by polyvinyl alcohol[J]. Iranian Polymer Journal, 2015, 24(1): 13-20.

[10]Zhang Y, Yang C, Zheng J, et al. Crystallization behavior of stable urea formaldehyde resin dispersed by polyvinyl alcohol[J]. Iranian Polymer Journal, 2015, 24(1): 13-20.

[11] Yousaf A M, Kim D W, Kim J K, et al. Novel fenofibrate-loaded gelatin microcapsules with enhanced solubility and excellent flowability: Preparation and physicochemical characterization[J]. Powder Technology, 2015, 275: 257-262.

[12]Wang D, Chen M, Wang C, et al. Synthesis of carbon microspheres from urea formaldehyde resin[J]. Materials Letters, 2011, 65(7): 1069-1072.

[13] Siimer K, Kaljuvee T, Christjanson P. Thermal behaviour of urea-formaldehyde resins during curing[J]. Journal of thermal analysis and calorimetry, 2003, 72(2): 607-617.

[14]Hordijk A C, Leurs O. Gun Barrel Erosion-Comparison of Conventional and LOVA Gun Propellants[J]. Journal of pressure vessel technology, 2006, 128(2): 246-250.

[15] Chen Y, Song Q, Wang J. Thermochemical Erosion of Propellant with Nanometer Additives [J]. Acta Armamentarii, 2007, 28(3): 329-331 